\title{
A CORE EXISTENCE THEOREM FOR GAMES WITHOUT ORDERED PREFERENCES
}

\author{
BY KIM C. BORDER ${ }^{1}$
}

\section{INTRODUCTION}

TO A LARGE EXTENT the cooperative theory of games has an altogether different appearance from the noncooperative theory. The noncooperative theory generally deals with games in either extensive form or normal form, while the cooperative theory is usually described in characteristic function form. One of the central concepts in the cooperative theory is that of the core, which is the set of utility allocations which no coalition can improve upon. This notion of the core and of the characteristic function form of a game depends heavily on the existence of a utility representation for players' preferences. Recently Gale and Mas-Colell [3] and Shafer and Sonnenschein [6] have proven theorems on the existence of a Nash equilibrium for noncooperative games in normal form in which the players' preferences over strategy vectors are not necessarily complete or transitive and so may fail to have a utility representation. Thus it might appear that the noncooperative theory is applicable in environments where the cooperative theory is not. In order to formulate theorems in the cooperative theory of games which can be applied to environments in which players may have nonordered preferences, the characteristic function must be reformulated in terms of physical outcomes as opposed to utility outcomes. The players' preferences can then be expressed in terms of the physical outcomes without the use of a utility function.

Characterizing a game in terms of the physical outcomes that a coalition can enforce for its members is not a new idea. This is implicitly the model used in discussing the core of a market economy. More generally, the description of a game in "outcome-characteristic function form" specifies the following. Each player $i \in N$ has a set $S_{i}$ of personal outcomes that might potentially obtain. The description of a personal outcome describes all the aspects of the outcome which are relevant to player $i$. Then player $i$ 's preferences depend only on which personal outcome obtains. The characteristic function describes which vectors of personal outcomes a coalition can somehow guarantee for its members. In general, this set depends on the outcomes obtaining for players outside the coalition. Thus for each coalition $B$ there is a correspondence $F^{B}: \prod_{i \in N} S_{i} \rightarrow \rightarrow \prod_{i \in B} S_{i}$ that describes the set of feasible outcome vectors for the coalition. Finally there is a set $F \subset \prod_{i \in N} S_{i}$ of jointly feasible outcome vectors. This set $F$ may be different from $\left\{x: x \in F^{N}(x)\right\}$.

A coalition $B$ blocks an outcome vector $x$ if there is some $y^{B} \in F^{B}(x)$ such that each member of $B$ prefers $y^{B}$ to $x$. The set of all jointly feasible outcome vectors that are unblocked by any coalition is the core of the game.

An example is given by a pure exchange economy. A personal outcome for player $i$ consists of a consumption vector. Player $i$ 's preferences depend only on his own consumption. The set $S_{i}$ is just player $i$ 's consumption set. For each coalition $B, F^{B}$ is the set of allocations of $B$ 's aggregate endowment among its members. In this example $F^{B}$ does not depend on the consumption of players outside of $B$ and $F=F^{N}$. The core of the game defined this way coincides with the usual definition of the core of the market.

The theorem below provides a set of sufficient conditions on a game which guarantee the nonemptiness of its core, even if preferences are not required to be transitive or complete. The major requirement is a kind of balancedness condition. The proof of the theorem is followed by a discussion of the difference between the notion of balancedness used here and Scarf's [5] balancedness condition. The theorem is then used to prove a

\footnotetext{
${ }^{1}$ I am indebted to Michael Maschler, Roger Myerson, Bezalel Peleg, Jim Snyder, and Nicholas Yannelis for insightful comments.
} 
new result on the nonemptiness of the core of a coalitional production economy without ordered preferences.

\section{NOTATION AND DEFINITIONS}

A game $G$ is a tuple $\left(N,\left(S_{i}\right),\left(F^{B}\right), F,\left(P_{i}\right)\right)$ where $N=\{1, \ldots, n\}$ denotes the set of players. Each player $i$ has a set $S_{i}$ of personal outcomes potentially available to him. Preferences are represented by a correspondence $P_{i}: S_{i} \rightarrow \rightarrow S_{i}$. The interpretation is that $z_{i} \in P_{i}\left(x_{i}\right)$ means that $z_{i}$ is strictly preferred to $x_{i}$. Note that $i$ 's preferences depend only on his personal outcome. For each coalition $B \subset N$ let $S^{B}=\prod_{i \in B} S_{i}$ and set $S=\prod_{i \in N} S_{i}$. Given the outcome vector $x$, the set of outcomes which are jointly feasible for members of coalition $B$ is denoted $F^{B}(x) \subset S^{B}$. The set of all jointly feasible outcome vectors is denoted by $F \subset S$.

The core of $G=\left(N,\left(S_{i}\right),\left(F_{B}\right), F,\left(P_{i}\right)\right)$ is the set of all $x \in S$ satisfying

$$
x \in F \text {, }
$$

and

$$
\text { there is no } B \subset N \text { and } z^{B} \in F^{B}(x) \text { satisfying } z_{i}^{B} \in P_{i}\left(x_{i}\right) \text { for all } i \in B \text {. }
$$

Let $\beta$ be a family of subsets of $N$. For each $i \in N$, let $\beta(i)=\{B \in \beta: i \in B\}$. The family $\beta$ is balanced if there are scalars $\left\{\lambda_{B} \geqslant 0: B \in \beta\right\}$ (called balancing weights) such that for each $i \in N$,

$$
\sum_{B \in \beta(i)} \lambda_{B}=1 .
$$

Let $a^{1}, \ldots, a^{n}$ be an affinely independent set of vectors and set $m_{B}=(1 /|B|) \sum_{i \in B} a^{i}$. Then $\beta$ is a balanced family if and only if $m_{N} \in \operatorname{co}\left\{m_{B}: B \in \beta\right\}$, where co denotes the convex hull. (If $m_{N}=\sum_{B \in \beta} \alpha_{B} m_{B}$ then balancing weights may be found by setting $\lambda_{B}=(n /|B|) \alpha_{B}$.)

The game $G$ is balanced if whenever $\beta$ is a balanced family with balancing weights $\left\{\lambda_{B}\right\}$, and $x^{B} \in F^{B}(z)$ for each $B \in \beta$, then $x \in F$ where $x_{i}=\sum_{B \in \beta(i)} \lambda_{B} x_{i}^{B}$.

\section{NONEMPTINESS OF THE CORE}

THEOREM: Let $G=\left(N,\left(S_{i}\right),\left(F^{B}\right), F,\left(P_{i}\right)\right)$ be a game satisfying:

1. For each $i, S_{i}$ is a nonempty convex subset of $\mathbb{R}^{k_{i}}$.

2. For each nonempty $B \subset N, F^{B}: S \rightarrow \rightarrow S^{B}$ is a continuous correspondence with compact values and for each $i, F^{\{i\}}$ is nonempty-valued.

3. $F$ is compact and convex.

4. For each $i,(a) P_{i}$ has open graph in $S_{i} \times S_{i} ;(b) x_{i} \notin \operatorname{co~} P_{i}\left(x_{i}\right)$.

5. $G$ is balanced.

Then $G$ has a nonempty core.

PROOF: The first claim is that we may without loss of generality assume that $P_{i}\left(x_{i}\right)$ is convex and $x_{i} \notin P_{i}\left(x_{i}\right)$. If not, we can replace $P_{i}: S_{i} \rightarrow \rightarrow S_{i}$ by $\tilde{P}_{i}: S_{i} \rightarrow \rightarrow S_{i}$ where $\tilde{P}_{i}(x)=$ co $P_{i}(x)$. Then $\tilde{P}_{i}$ will still have open graph and by Assumption $2 \mathrm{~b}, x_{i} \notin \tilde{P}_{i}(x)$. If $x$ belongs to the core of the game $\tilde{G}$ defined by replacing $P_{i}$ by $\tilde{P}_{i}$ for each $i$, then $x$ must also belong to the core of $G$. This is because if $z^{B} \in F^{B}(x)$ implies $z_{i}^{B} \notin \tilde{P}_{i}(x)$, since $P_{i}(x) \subset \tilde{P}_{i}(x)$, we have $a$ fortiori that $z_{i}^{B} \notin P_{i}(x)$. So for the rest of the proof we will assume $P_{i}(x)$ is convex.

Begin the proof of the theorem by defining $v_{i}: S_{i} \times S_{i} \rightarrow \mathbb{R}_{+}$by

$$
v_{i}\left(z_{i}, x_{i}\right)=\operatorname{distance}\left[\left(x_{i}, z_{i}\right),\left(\mathrm{Gr} P_{i}\right)^{c}\right] \text {, }
$$

where $\operatorname{Gr} P_{i}=\left\{\left(x_{i}, z_{i}\right)=z_{i} \in P_{i}\left(x_{i}\right)\right\}$, the graph of $P_{i}$. (This construction is due to Shafer and Sonnenschein [6].) Each $v_{i}$ is continuous (as Gr $P_{i}$ is open), and $v_{i}\left(z_{i}, x_{i}\right)>0$ if and 
only if $z_{i} \in P_{i}\left(x_{i}\right)$. The function $v_{i}$ acts as a "pseudo-utility" for $\boldsymbol{P}_{i}$, and possesses the following important property. Suppose $v_{i}\left(z_{i}^{k}, x_{i}\right) \geqslant w$ for $k=1, \ldots, p$. Let $z_{i}$ be a convex combination of $z_{i}^{1}, \ldots, z_{i}^{k}$. Then $v_{i}\left(z_{i}, x_{i}\right) \geqslant w$, i.e., $v_{i}$ is quasi-concave in its first argument. The proof of this may be found in the Appendix.

For each $B \subset N$ set

$$
V^{B}(x)=\left\{w \in \mathbb{R}^{N}: \exists z^{B} \in F^{B}(x) \forall i \in B, w_{i} \leqslant v_{i}\left(z_{i}^{B}, x_{i}\right)\right\} .
$$

If $i \notin B$, then $w \in V^{B}(x)$ places no restriction on $w_{i}$. Thus $x$ is in the core if and only if $x \in F$ and $\bigcup_{B \in N} V^{B}(x) \cap \mathbb{R}_{++}^{N}=\varnothing$. Note that $V^{B}(x)$ may be empty, but each $V^{\{i\}}(x)$ is nonempty. Since $\{\{i\}: i \in N\}$ is a balanced family with weights $\lambda_{\{i\}}=1$, and since each $F^{\{i\}}$ is nonempty, balancedness of $G$ implies that $F$ is nonempty.

The sets $V^{B}(x)$ are analogues of the characteristic function of a game without side payments and the arguments of Shapley [7] and Ichiishi [4] may be applied. The following line of argument is similar to Ichiishi [4].

Since each $v_{i}$ is continuous and since $F$ is compact and each $F^{B}$ is continuous and compact-valued, there is some $M \geqslant 0$ such that for all $x \in F$, and $z^{B} \in F^{B}(x), v_{i}\left(z_{i}^{B}, x_{i}\right) \leqslant M$ for all $i \in B$. Put $a^{i}=-M n e^{i} \in \mathbb{R}^{n}$ (where $e^{i}$ is the $i$ th unit coordinate vector of $\mathbb{R}^{n}$ ) and set $\Delta=\operatorname{co}\left\{a^{i}: i \in N\right\}$. For each $B \subset N$, set $m_{B}=(1 /|B|) \sum_{i \in B} a^{i}$.

For each $y \in \Delta$ set

$$
\tau(y, x)=\max \left\{t>0: y+t(1, \ldots, 1) \in \bigcup_{B \subset N} V^{B}(x)\right\},
$$

and put $w(y, x)=y+\tau(y, x)(1, \ldots, 1)$. Note that $\tau(y, x) \leqslant M(n+1)$ and $w(y, x) \leqslant$ $M(1, \ldots, 1)$. Since $v_{i}$ is always nonnegative, $V^{\{k\}}(x)$ always includes $\left\{w: w_{k} \leqslant 0\right\}$. Suppose that some $w_{k}(y, x)<0$; then $w(y, x)=y+\tau(y, x)(1, \ldots, 1)$ is in the interior of $V^{\{k\}}(x)$, which contradicts the definition of $\tau$. Thus $w(y, x) \geqslant 0$.

The next step is to show that if $x \in F$ and $w(y, x) \leqslant 0$, then $x$ is in the core. Suppose not. Then for some $z^{B} \in F^{B}(x), z_{i}^{B} \in P_{i}(x)$ for all $i \in B$, so $v_{i}\left(z_{i}^{B}, x_{i}\right)>0$ for all $i \in B$. Thus there is a $w \in V^{B}(x)$ with $w>0$. But then $y+\tau(y, x)(1, \ldots, 1)=w(y, x) \leqslant 0<w$ is in the interior of $V^{B}(x)$, which contradicts the definition of $\tau$. Note that $w(y, x) \leqslant 0$ implies that $w(y, x)=0($ as $w(y, x) \geqslant 0)$ and so $y=m_{N}$.

Thus the search for an element of the core has been reduced to the following problem: Find $x \in F$ and $y \in \Delta$ such that $w(y, x) \leqslant 0$. To this end make the following constructions. Define

$$
E(x, y)=\{z \in F: z \text { minimizes distance }[v(\cdot, x),\{w: w \geqslant w(y, x)\}]\}
$$

where the $i$ th component of $v(x, y)$ is $v_{i}\left(x_{i}, y_{i}\right)$. Define $\gamma, \mu: F \times \Delta \rightarrow \rightarrow F \times \Delta$ by

$$
\gamma(x, y)=\{x\} \times \operatorname{co}\left\{m_{B}: w(y, x) \in V^{B}(x)\right\}
$$

and

$$
\mu(x, y)=\operatorname{co} E(x, y) \times\left\{m_{N}\right\} .
$$

The correspondences $\gamma$ and $\mu$ so defined satisfy the hypotheses of Theorem 6 of Fan [2]. The statement of Fan's theorem and the proof of this claim is given in the Appendix. It follows from Fan's theorem that there are $\bar{x}, \bar{y}, x^{*}, y^{*}$ satisfying

$$
(\bar{x}, \bar{y}) \in \mu\left(x^{*}, y^{*}\right) \cap \gamma\left(x^{*}, y^{*}\right) \text {. }
$$

In other words,

$$
\begin{aligned}
& \bar{x} \in \operatorname{co} E\left(x^{*}, y^{*}\right), \\
& \bar{y}=m_{N}, \\
& \bar{x}=x^{*}, \\
& \bar{y} \in \operatorname{co}\left\{m_{B}: w\left(y^{*}, x^{*}\right) \in V^{B}\left(x^{*}\right)\right\} .
\end{aligned}
$$


By (2) and (4), $\beta=\left\{B: w\left(y^{*}, x^{*}\right) \in V^{B}\left(x^{*}\right)\right\}$ is balanced. Let $\left\{\lambda_{B}\right\}$ be the associated balancing weights. By the definition of $V^{B}$, for each $B \in \beta$ there exists $z^{B} \in F^{B}\left(x^{*}\right)$ satisfying $w_{i}\left(y^{*}, x^{*}\right) \leqslant v_{i}\left(z_{i}^{B}, x_{i}^{*}\right)$ for all $i \in B$. Since the game is balanced, $z^{*} \in F$ where $z_{i}^{*}=$ $\sum_{B \in \beta(i)} \lambda_{B} z_{i}^{B}$. Since $z_{i}^{*}$ is a convex combination of the $z_{i}^{B}$ for $B \in \beta(i)$ and $v_{i}\left(z_{i}^{B}, x_{i}^{*}\right) \geqslant$ $w_{i}\left(y^{*}, x^{*}\right)$, it follows by quasi-concavity that $v_{i}\left(z_{i}^{*}, x_{i}^{*}\right) \geqslant w_{i}\left(y^{*}, x^{*}\right)$.

By (1) and (3), $x^{*} \in \operatorname{co} E\left(x^{*}, y^{*}\right)$. Since $z^{*} \in F$ and $v\left(z^{*}, x^{*}\right) \geqslant w\left(y^{*}, x^{*}\right)$ it follows from the definition of $E$ that if $z \in E\left(x^{*}, y^{*}\right)$, then $v\left(z, x^{*}\right) \geqslant w\left(y^{*}, x^{*}\right)$. Suppose that $w_{i}\left(y^{*}, x^{*}\right)>0$ for some $i$. Then for all $z \in E\left(x^{*}, y^{*}\right), v_{i}\left(z_{i}, x_{i}^{*}\right)>0$ as well, so that $z_{i} \in P_{i}\left(x_{i}^{*}\right)$. Thus $x^{*} \in \operatorname{co} E\left(x^{*}, y^{*}\right)$ implies that $x_{i}^{*} \in \operatorname{co} P_{i}\left(x_{i}^{*}\right)$, a contradiction. Thus $w_{i}\left(y^{*}, x^{*}\right) \leqslant 0$. Also since $F$ is convex and $E\left(x^{*}, y^{*}\right) \subset F$, it follows that $x^{*} \in F$. Thus $x^{*}$ is in the core.

Q.E.D.

\section{THE BALANCEDNESS CONDITION}

The balancedness condition in this paper is due to Boehm [1] and implies Scarf's [5] condition. Scarf worked with games in utility-characteristic function form, but the natural translation of his condition in the current framework is as follows. Let $x \in S$ and let $\pi^{B}(x)$ denote the projection of $x$ on $S^{B}$. Scarf's condition is that if $\pi^{B}(x) \in F^{B}(z)$ for all $B$ in some balanced family $\beta$, then $x \in F$. This follows from the balancedness conditions used here by simply noting that $x_{i}=\sum_{B \in \beta(i)} \lambda_{B} \pi^{B}(x)_{i}$, where $\left\{\lambda_{B}\right\}$ is a collection of balancing weights for $\beta$. Scarf's condition is inadequate to prove the theorem with the techniques used above, so that this theorem cannot be viewed as a strict generalization of Scarf's theorem.

\section{THE CORE OF A COALITIONAL PRODUCTION ECONOMY}

The above theorem has as an immediate consequence the following result for coalitional production economies. The model adopted is essentially that of Boehm [1]. The commodity space is $\mathbb{R}^{m}$. Each agent $i$ has a consumption set $S_{i} \subset \mathbb{R}^{m}$, endowment $w^{i} \in S_{i}$, and preference correspondence $P_{i}: S_{i} \rightarrow \rightarrow S_{i}$. Each coalition $B$ has a production set $Y^{B} \subset \mathbb{R}^{m}$ and the aggregate production set is $Y \subset \mathbb{B}^{m}$. An allocation is an $\left(x^{1}, \ldots, x^{n}\right) \in \prod_{l \in N} S_{i}$ satisfying

$$
\sum_{i \in N} x^{i}-\sum_{i \in N} w^{i} \in Y
$$

Allocation $x=\left(x^{1}, \ldots, x^{n}\right)$ is blocked by coalition $B$ if there is some $z^{B}=\left(z^{i}\right)_{i \in B} \in S^{B}$ satisfying $\sum_{i \in B} z_{i}^{B}-\sum_{i \in B} w^{i} \in Y^{B}$ and for all $i \in B, z^{i} \in P_{i}\left(x^{i}\right)$. The core of the economy is the set of unblocked allocations.

Proposition (cf. Boehm [1]): Assume that (1) for each $i, S_{i}$ is closed, convex and bounded below; (2) for each $i, P_{i}$ has open graph and $x^{i} \notin \operatorname{co} P_{i}\left(x^{i}\right) ;(3)$ for each $i, 0 \in Y^{\{i\}}$; (4) for each $B \subset N, Y^{B}$ is closed; (5) $Y$ is closed and convex and $A Y \cap \mathbb{R}_{+}^{M}=\{0\}$, where $A Y$ denotes the asymptotic core of $Y$; (6) for each balanced family $\beta$ of coalitions with balancing weights $\left\{\lambda_{B}\right\}, \sum_{B \in \beta} \lambda_{B} Y^{B} \subset Y$. Then the core of the economy is nonempty.

PROOF: Set

$$
F^{B}(x)=\left\{z^{B} \in S^{B}: \sum_{i \in B} z_{i}^{B}-\sum_{i \in B} w^{i} \in Y^{B}\right\}
$$

and

$$
F=\left\{x \in S: \sum_{i \in N} x^{i}-\sum_{i \in N} w^{i} \in Y\right\} .
$$


Then $F$ is compact by (1) and (4) and convex by (4). It is easily seen that (6) implies that the resulting game is balanced. Condition (3) implies that each $F^{\{i\}}$ is nonempty. Thus by the theorem above, the core is nonempty.

Q.E.D.

This theorem differs from Boehm's in two respects. First, preferences are not assumed to be transitive or complete. This is traded for the additional assumption needed here that the aggregate production set $Y$ is convex.

\section{California Institute of Technology}

Manuscript received July, 1983; revision received January, 1984.

\section{APPENDIX}

\section{Quasi-concavity of $v_{i}$ in its First Argument}

For convenience, the common subscript $i$ will be omitted. Let $v\left(z^{k}, x\right) \geqslant w, k=1, \ldots, p$, and let $z=\sum_{k=1}^{p} \lambda_{k} z^{k}$ be a convex combination of $z^{1}, \ldots, z^{p}$. Then $v(z, x) \geqslant w$.

If $w \leqslant 0$, the result is trivial. If $w>0$, let $N_{w}\left(x, z^{k}\right)$ be an open ball of radius $w$ about $\left(x, z^{k}\right)$. From the definition of $v, N_{w}\left(x, z^{k}\right) \subset \mathrm{Gr} P$, for all $k=1, \ldots, p$. Let $\left(x^{\prime}, z^{\prime}\right) \in N_{w}(x, z)$. Then $\mid\left(x^{\prime}-x\right.$, $\left.z^{\prime}-z\right) \mid<w$ so

$$
\left(x+\left(x^{\prime}-x\right), z^{k}+\left(z^{\prime}-z\right)\right) \in N_{w}\left(x, z^{k}\right) \subset \operatorname{Gr} P .
$$

Thus $z^{k}+z^{\prime}-z \in P\left(x^{\prime}\right)$. Since we may assume that $P\left(x^{\prime}\right)$ is convex,

$$
z^{\prime}=\sum_{k=1}^{p} \lambda_{k}\left(z^{k}+z^{\prime}-z\right) \in P\left(x^{\prime}\right)
$$

so that $\left(x^{\prime}, z^{\prime}\right) \in \operatorname{Gr} P$. Thus $N_{w}(x, z) \subset \mathrm{Gr} P$, so $v(z, x) \geqslant w$.

\section{The Correspondences $\gamma$ and $\mu$ Satisfy the Hypotheses of Fan's Theorem}

The proof relies on the following result which is a special case of a theorem of Fan [2, Theorem 6]. Let $K \subset \mathbb{R}^{n}$ be compact and convex, and let $\gamma, \mu: K \rightarrow \rightarrow \mathbb{R}^{n}$ be upper hemi-continuous with nonempty compact convex values. Suppose that for each $x \in K$ there exist three points $y \in K$, $u \in \gamma(x), v \in \mu(x)$ and a real number $\lambda>0$ such that $y=x+\lambda(u-v)$. Then there is a $z \in K$ such that $\gamma(z) \cap \mu(z) \neq \varnothing$.

It is straightforward to verify that $\gamma$ and $\mu$ are upper hemi-continuous with nonempty compact convex values. It is harder to see that for every $(x, y) \in F \times \Delta$, there exist $\left(x^{\prime}, y^{\prime}\right) \in \mu(x, y),\left(x^{\prime \prime}, y^{\prime \prime}\right) \subset$ $\gamma(x, y)$ and $\bar{\lambda}>0$ satisfying

$$
\left[(x, y)+\bar{\lambda}\left(x^{\prime}, y^{\prime}\right)-\left(x^{\prime \prime}, y^{\prime \prime}\right)\right] \in F \times \Delta .
$$

The argument is virtually identical to one used by Ichiishi [4] with only slightly different correspondences. Put $x^{\prime \prime}=x, y^{\prime}=m_{N}$, and choose any $x^{\prime} \in \operatorname{co} E(x, y)$. Then

$$
x+\lambda\left(x^{\prime}-x^{\prime \prime}\right)=(1-\lambda) x+\lambda x^{\prime} \in F
$$

for any $\lambda \in[0,1]$. Let $B \subset N=\left\{i: y_{t}<0\right\}$. Set $\Gamma^{B}(x)=\left\{\tilde{y} \in \Delta: w(\tilde{y}, x) \in V^{B}(x)\right\}$. It is shown below that co $\left\{a^{i}: i \in B\right\} \subset \bigcup_{C \subset B} \Gamma^{C}(x)$. Given this, choose $C \subset B$ so that $y \in \Gamma^{C}(x)$. Put $y^{\prime \prime}=m_{C}$. Then $\left(x^{\prime \prime}, y^{\prime \prime}\right) \in$ $\gamma(x, y)$. For $\lambda \in[0,1]$, define $y^{\lambda}=y+\lambda\left(y^{\prime}-y^{\prime \prime}\right)=y+\lambda\left(m_{N}-m_{C}\right)$. Then

$$
\sum_{i=1}^{n} y_{i}^{\lambda}=\sum_{i=1}^{n} y_{i}+\lambda\left(\sum_{i=1}^{n} m_{N i}-\sum_{i=1}^{n} m_{C_{l}}\right)=-M n+\lambda(-M n+M n)=-M n
$$

and if $\bar{\lambda}$ is small enough, $y^{\bar{\lambda}} \leqslant 0$ so $y^{\bar{\lambda}} \in \Delta$.

The argument that $\operatorname{co}\left\{a^{i} \mid i \in B\right\} \subset \bigcup_{C \subset B} \Gamma^{C}(x)$ for all $B$ is due to Shapley [7]. If $B=N$ this just says that $w(y, x) \in V^{C}(x)$ for some $C$, so suppose that $B \neq N$, so that $|B|<n$. Let $y \in \Gamma^{C}(x) \cap$ co $\left\{a^{i}: i \in B\right\}$. We need to show that $C \subset B$. Since $y \in \Gamma^{C}(x), w(y, x) \in V^{C}(x)$, so $w_{j}(y, x)=$ $y_{j}+\tau(y, x) \leqslant M$ for all $j \in C$. But $\sum_{J \in B} y_{J}=-M n$, and for some $k \in B, y_{k}$ is less than equal the average of the $y_{j}$ 's for $j \in B$, so $y_{k} \leqslant-M n /|B|<-M$ as $|B|<n$. But $w(y, x) \geqslant 0$ so $y_{k}+\tau(y, x) \geqslant 0$, so $\tau(y, x)>M$. 
This and $y_{j}+\tau(y, x) \leqslant M$ for $j \in C$ imply that $y_{j}<0$ for all $j \in C$. Since $y \in \operatorname{co}\left\{a^{i}: i \in B\right\}$ and $y_{J}<0$ for $j \in C$, it follows that $C \subset B$.

\section{REFERENCES}

[1] BоEнм, V.: "The Core of an Economy with Production," Review of Economic Studies, 41(1974), 429-436.

[2] FAN, K.: "Extensions of Two Fixed Point Theorems of F. E. Browder," Mathematische Zeitschrift, 112(1969), 234-240.

[3] Gale, D., AND A. MAs-Colell: "An Equilibrium Existence Theorem for a General Model without Ordered Preferences," Journal of Mathematical Economics, 2(1975), 9-15.

[4] ICHIISHI, T.: "A Social Coalitional Equilibrium Existence Theorem," Econometrica, 49(1981), 369-377.

[5] SCARF, H.: "The Core of an N Person Game," Econometrica, 35 (1967), 50-69.

[6] ShAFER, W., AND H. SONNENSCHEIN: "Equilibrium in Abstract Economics Without Ordered Preferences," Journal of Mathematical Economics, 2(1975), 345-348.

[7] Shapley, L. S.: "On Balanced Games Without Side Payments," in Mathematical Programming, ed. by T. C. Hu and S. M. Robinson. New York: Academic Press, 1973. 